\title{
Erratum zu: Vorwort der Herausgeber
}

\author{
Timo Schmid • Markus Zwick
}

Online publiziert: 22. Mai 2019

(C) Springer-Verlag GmbH Germany, part of Springer Nature 2019

\section{Erratum zu:}

AStA Wirtsch Sozialstat Arch 2019

https://doi.org/10.1007/s11943-019-00244-w

Durch einen Produktionsfehler wurde in der Originalveröffentlichung versehentlich nur einer der beiden Autoren genannt.

Die Autoren des Vorworts sind Timo Schmid und Markus Zwick.

Der Verlag bittet, diesen Fehler zu entschuldigen.

Die Online-Version des Originalartikels ist unter https://doi.org/10.1007/s11943-019-00244-w zu finden.

\section{T. Schmid}

Institut für Statistik und Ökonometrie, Freie Universität Berlin, Berlin, Deutschland

M. Zwick $(\bowtie)$

Institut für Forschung und Entwicklung in der Bundesstatistik, Statistisches Bundesamt Wiesbaden, Wiesbaden, Deutschland

E-Mail: markus.zwick@destatis.de 